\title{
xCELLigence system for real-time label-free monitoring of growth and viability of cell lines from hematological malignancies
}

This article was published in the following Dove Press journal:

OncoTargets and Therapy

12 June 2014

Number of times this article has been viewed

\author{
Jordi Martinez-Serra' \\ Antonio Gutierrez' \\ Saúl Muñoz-Capó' \\ María Navarro-Palou' \\ Teresa Ros' \\ Juan Carlos Amat ${ }^{\prime}$ \\ Bernardo Lopez' \\ Toni F Marcus' \\ Laura Fueyo ${ }^{2}$ \\ Angela G Suquia ${ }^{2}$ \\ Jordi Gines ${ }^{3}$ \\ Francisco Rubio' \\ Rafael Ramos ${ }^{4}$ \\ Joan Besalduch' \\ 'Department of Hematology, \\ ${ }^{2}$ Department of Clinical Analysis, \\ ${ }^{3}$ Department of Pharmacy, \\ ${ }^{4}$ Department of Pathology, University \\ Hospital Son Espases, Palma de \\ Mallorca, Balearic Islands, Spain
}

Correspondence: Jordi Martinez-Serra Department of Hematology, University Hospital of Son Espases, Crta Valldemossa n-79, Palma de

Mallorca, Baleares, Spain

Tel +34 87I 205000 , ext 76267

Email jorgej.martinez@ssib.es

\begin{abstract}
The xCELLigence system is a new technological approach that allows the real-time cell analysis of adherent tumor cells. To date, xCELLigence has not been able to monitor the growth or cytotoxicity of nonadherent cells derived from hematological malignancies. The basis of its technology relies on the use of culture plates with gold microelectrodes located in their base. We have adapted the methodology described by others to xCELLigence, based on the pre-coating of the cell culture surface with specific substrates, some of which are known to facilitate cell adhesion in the extracellular matrix. Pre-coating of the culture plates with fibronectin, compared to laminin, collagen, or gelatin, significantly induced the adhesion of most of the leukemia/lymphoma cells assayed (Jurkat, L1236, KMH2, and K562). With a fibronectin substrate, nonadherent cells deposited in a monolayer configuration, and consequently, the cell growth and viability were robustly monitored. We further demonstrate the feasibility of xCELLigence for the real-time monitoring of the cytotoxic properties of several antineoplastic agents. In order to validate this technology, the data obtained through real-time cell analysis was compared with that obtained from using the 3-(4,5-dimethylthiazol-2-yl)-2,5-diphenyltetrazolium bromide method. This provides an excellent label-free tool for the screening of drug efficacy in nonadherent cells and discriminates optimal time points for further molecular analysis of cellular events associated with treatments, reducing both time and costs.
\end{abstract}

Keywords: real-time cell analysis, drug discovery, leukemia, lymphoma

\section{Introduction}

Drug discovery is still an expensive and inefficient process in cancer research. ${ }^{1}$ Despite the considerable progress that has been made in preclinical model development and therapeutic targets identification, new drugs are needed. The process of drug discovery involves many stages, such as the identification of new candidates, synthesis, characterization, screening, and assays for therapeutic efficacy. In this context, cell-based assays are an important part of the preclinical drug development procedure. The emergence of new technologies that facilitate this procedure is a priority. The xCELLigence system (ACEA Biosciences, Inc., San Diego, CA, USA) is a new technological approach that makes the real-time cell analysis (RTCA) of a cell culture possible. This new concept has just emerged as an interesting method based on the use of culture plates (E-Plates) with gold microelectrodes in their base. These electrodes are connected to a computer that measures the impedance differences within an electrical circuit. These differences are converted into cell index (CI), a value that may be influenced by several parameters, such as cell number, cell size, cell-substrate, or cell-cell attachment., ${ }^{2,3}$ Therefore, xCELLigence uses impedance 
measurements for the real-time monitoring of cell growth and death. To date, the xCELLigence system has mostly been used to monitor adherent cells' behavior. Most leukemia or lymphoma cells usually grow in liquid media or suspension and they are unable to attach onto the cell culture wells' surface, where the electrodes are located, so changes within the electric circuit cannot be properly measured. So far, with the xCELLigence technology, there has only been one report describing a system for measuring cell adhesion of cell lines derived from hematological malignancies. ${ }^{4}$ With this in mind, we have taken advantage of the strategy described by others, ${ }^{4-7}$ based on the addition of several coating substrates such as fibronectin, collagen, gelatin, and/or laminin. These substrates are known to facilitate cell attachment of nonadherent cells. In this manuscript, we show the feasibility of this approach for several hematological derived cell lines. Pre-coating of E-Plates with fibronectin facilitates the adhesion of suspension-type cells allowing them to be monitored. At the same time, we determined whether xCELLigence is also capable of reliably measuring the death rate of leukemia/lymphoma cells in response to several antineoplastic drugs. The real-time follow-up instead of endpoint experiments may give us a better understanding of cell behavior in response to a drug or signaling molecule (hormones, cytokines, etc). Moreover, avoiding the use of any external label for the monitoring of cell dynamics means a minimal interference with experimental conditions.

\section{Methods}

\section{Drugs and cell culture}

Trabectedin $(500 \mathrm{nM})$, oxaliplatin $(10 \mathrm{mM})$, bendamustine (10 mM), Fas ligand (FasL; 500 ng/mL), cisplatin (10 mM), doxorubicin $(10 \mathrm{mM})$, and gemcitabine $(10 \mathrm{mM})$ were prepared as stock solutions dissolved in double-distilled sterile water. Before use, the stock solution was re-diluted in double-distilled sterile water to the desired concentrations. We included various cell lines derived from hematological malignancies: T cell acute lymphoblastic leukemia (Jurkat), Hodgkin's lymphoma (L1236 and KMH2), chronic myeloid leukemia in blast crisis (K562), and acute myeloblastic leukemia (U937) obtained from the American Type Culture Collection (Manassas, VA, USA) or the German Collection of Microorganisms and Cell Cultures (DSMZ; Braunschweig, Germany). Cells (10,000-240,000 cells/well) were grown in Roswell Park Memorial Institute (RPMI) 1640, supplemented with $2 \mathrm{mM}$ glutamine, 10\% (volume/volume) fetal calf serum, 100 units $/ \mathrm{mL}$ penicillin, $100 \mu \mathrm{g} / \mathrm{mL}$ streptomycin, and $0.25 \mu \mathrm{g} / \mathrm{mL}$ amphotericin $\mathrm{B}$ in $5 \% \mathrm{CO}_{2}$.
Tissue culture medium and supplements were purchased from Sigma-Aldrich Co, (St Louis, MO, USA). Integrin $\alpha 4$ antibody (VLA-4; C-20) was purchased from Santa Cruz Biotechnology Inc. (Dallas, TX, USA).

\section{Fibronectin, laminin collagen, and gelatin coating}

Fibronectin $(170 \mu \mathrm{g} / \mathrm{mL}$, double-distilled sterile water), gelatin $(0.2 \%$ volume per weight, double-distilled sterile water), collagen $(170 \mu \mathrm{g} / \mathrm{mL}$, double-distilled sterile water and $0.1 \mathrm{M}$ acetic acid), and laminin $(170 \mu \mathrm{g} / \mathrm{mL})$ were purchased from Sigma-Aldrich Co, and prepared as working solutions following the manufacturer's instructions. For precoating purposes, the well plates were incubated with $40 \mu \mathrm{L}$ of the working solution for each substrate. After 1 hour of incubation, the supernatant was removed and the wells were washed three times with $100 \mu \mathrm{L}$ phosphate-buffered saline $(1 \times)$. Once coated, depending on the experiment, between 40,000 and 240,000 cells were seeded.

\section{xCELLigence system: $\mathrm{Cl}$ and baseline $\mathrm{Cl}(\mathrm{B}-\mathrm{Cl})$ calculation}

The RTCA instrument is composed of a RTCA impedance analyzer, a computer with RTCA software for controlling the system operation, a 96-well electronic microtiter plate (E-Plate) and the RTCA station, which accommodates the E-Plate and it is placed inside the tissue culture incubator. The presence of cell culture medium or buffer and the application of a low voltage create an electric field between the electrodes, which can be impeded by cell presence. The electronic readout of cell-sensor impedance is displayed in real-time as CI, a value directly influenced by cell attachment, spreading, and/or cell proliferation. The $\mathrm{CI}$ value at each time point is defined as $R n-R b / R b$, where $R n$ is the cell-electrode impedance of the well with the cells and $R b$ is the background impedance of the well with only medium.

Fifty microliters of cell culture medium was added to each well for the impedance background measurement. After adding the cells and/or drugs, the final volume was $200 \mu \mathrm{L}$. The E-Plates were incubated at $37^{\circ} \mathrm{C}$ with $5 \%$ $\mathrm{CO}_{2}$ and monitored on the RTCA system at 5-minute time intervals for up to 72 hours with or without treatment. For data analysis, the B-CI is determined by subtracting the CI for a cell-containing well from the CI of a well with only culture media. To facilitate the statistical evaluation of the results, we performed at least four repeats of each experimental condition as recommended in the technical manual of xCELLigence. 


\section{MTT assay}

MTT assay is based on the reduction of MTT, a yellow tetrazole (3-[4,5-dimethylthiazol-2-yl]-2,5-diphenyltetrazolium bromide), by mitochondrial enzymes to form a purple formazan that is measured by spectrophotometric methods. MTT not only determines cell count and cell proliferation, but it can also be used for determining the cytotoxicity of various substances that would either inhibit or stimulate cell growth and propagation. Once the RTCA experiment was finished, we added $10 \mu \mathrm{L}$ of the MTT $(5 \mathrm{mg} / \mathrm{mL})$ stock solution to each well. After 4 hours at $37^{\circ} \mathrm{C}$, we measured the optical density (OD; $490 \mathrm{~nm}$ ) by spectrophotometry. The cell viability was plotted as OD percentage changes.

\section{Data analysis}

CI and MTT results were expressed as the mean \pm standard error of the mean, with at least three independent experiments. One-way analysis of variance (ANOVA) followed by Tukey's test was used (when required) for statistical evaluations of MTT values. The correlations between MTT (OD) and RTCA (B-CI) were calculated according to Spearman's rho. Differences were considered statistically significant at $P<0.05$.

\section{Results}

\section{Fibronectin pre-coating significantly increased Jurkat, $\mathrm{KMH} 2, \mathrm{~K} 562$, and U937 cells attachment onto the surface of the E-plate}

We first pre-coated the E-Plate surfaces (1 hour) with $6 \mu \mathrm{g}$ of fibronectin, collagen, laminin, or $0.2 \%$ volume per weight of gelatin. We then seeded Jurkat cells on the E-Plates. In the absence of a coating substrate, after 2 hours, the B-CI was almost 0 (mean B-CI: $0.1169 \pm 0.0658$ ). On the other hand, fibronectin, compared to the other substrates, allowed a significant and rapid increase of the B-CI (mean B-CI: $0.7106 \pm 0.0635$ ) (cell attaching stage), suggesting a more efficient adhesion induced by the presence of this coating substrate (Figure 1A). After 24 hours, the RTCA values for Jurkat cells reached a B-CI of $0.8849 \pm 0.0311$ for fibronectin, $0.1997 \pm 0.0378$ for collagen, $0.16720 \pm 0221$ for gelatin, and B-CI: $0.0570 \pm 0.0273$ for laminin (Figure 1A). We next incubated KMH2 cells, K562, and U937 in the presence of all these substrates. In Figure 1B, we show that under the same experimental conditions, almost all the leukemia/lymphoma cell lines tested showed a significant increased B-CI only in the presence of fibronectin. On the other hand, for U937 cells, even fibronectin failed to attach leukemia cells at the bottom of the well.

\section{The $\mathrm{Cl}$ is influenced by the cell number in the presence of fibronectin. Anti VLA-4 inhibits the $\mathrm{Cl}$ increase induced by fibronectin}

We seeded different amounts of Jurkat cells $(40,000$, $80,000,120,000$, and 240,000 cells/well) in the presence or absence of fibronectin (Figure 2A). As expected, we observed that the B-CI values, as calculated by xCELLigence, increased when a higher amount of cells were seeded (Figure 2). Therefore, the greater the number of cells deposited on the bottom of the well, the greater the $\mathrm{B}-\mathrm{CI}$ reading. We highlight that in the absence of fibronectin, the B-CI values were next to 0 . Moreover, in order to compare our results with a conventional method used for cell viability measurements, we carried out an MTT assay on the same plate, after 24 hours. We show that the MTT/ OD $(490 \mathrm{~nm})$ values correlated with those obtained in the RTCA (B-CI; $P=0.046$; Figure 2B). We also compared the values of the MTT assay when cells were incubated in the presence $(\mathrm{F}+)$ or absence $(\mathrm{F}-)$ of fibronectin. We could not detect significant differences between these two groups. This data suggests that fibronectin, under these experimental conditions, may not affect the viability/cell growth of the Jurkat cells. Finally, as adhesion to fibronectin occurs mainly through the VLA-4 integrin receptor, we incubated 50,000 Jurkat cells for 1 hour in the presence of different concentrations $(25,50$, and $100 \mu \mathrm{g} / \mathrm{mL})$ of an anti-VLA-4 antibody. The higher concentration of anti-VLA-4 corresponded with the higher inhibition of cell attachment to the bottom of the wells. A concentration of $100 \mu \mathrm{g} / \mathrm{mL}$ of anti-VLA-4 inhibited up to $60 \%$ of the CI (20 hours; $0.1704 \pm 0.0137$ ) reached when cells were incubated only in the presence of fibronectin (20 hours; $0.4004 \pm 0.0292$; Figure 2C). This data highlights the specificity of this integrin on the adherence of the leukemia cells on the RTCA plates.

\section{Fibronectin induces monolayer configuration/growth of Jurkat (leukemia) and LI 236 (lymphoma) cells}

We seeded 120,000 cells/well (Jurkat or L1236) in the presence/absence of fibronectin. We then monitored the cell growth characteristics. We showed a first 2-hour stage, in which, in the presence of fibronectin $(\mathrm{F}+)$, Jurkat and 

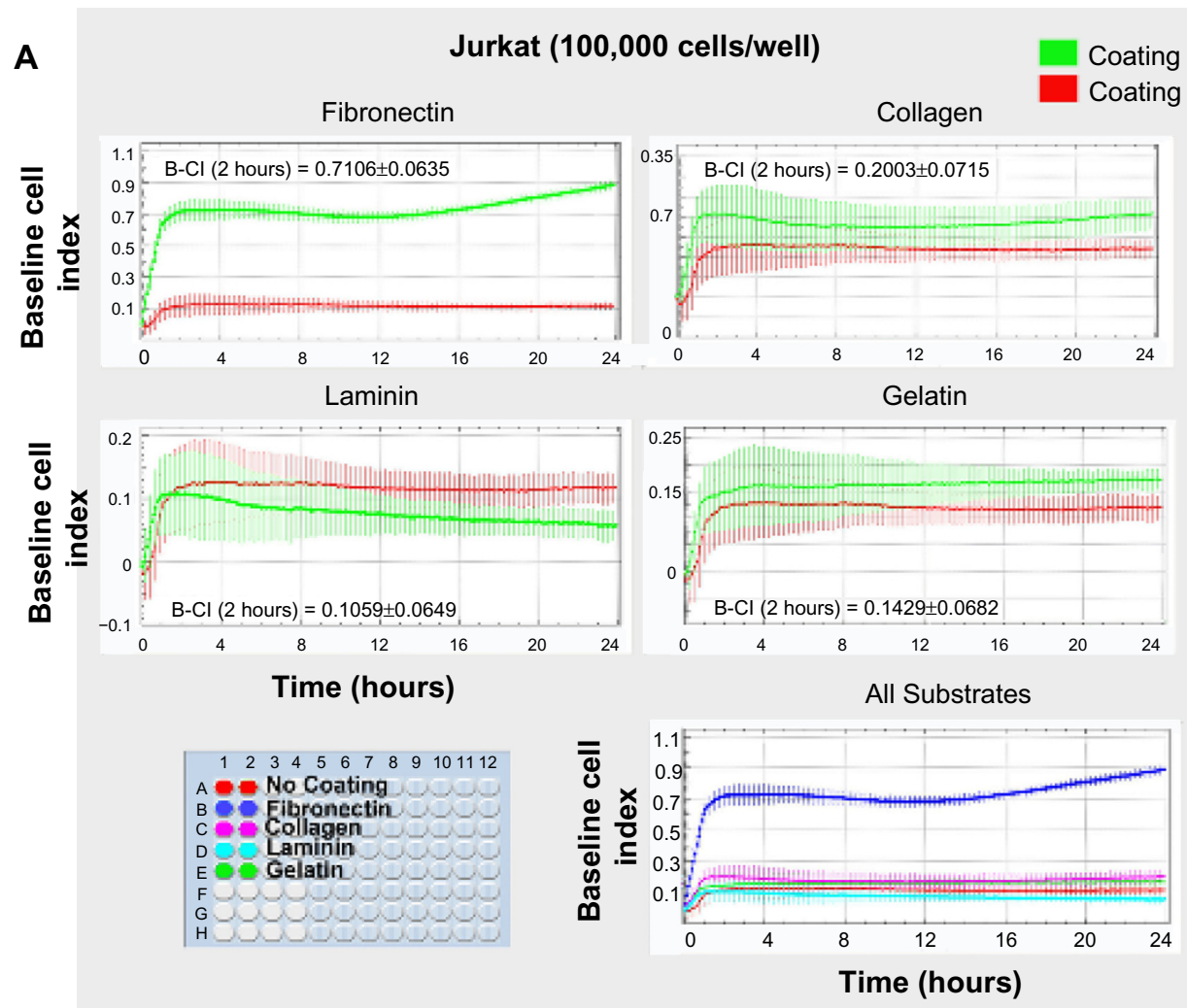

B

K562 (100,000 cells/well)

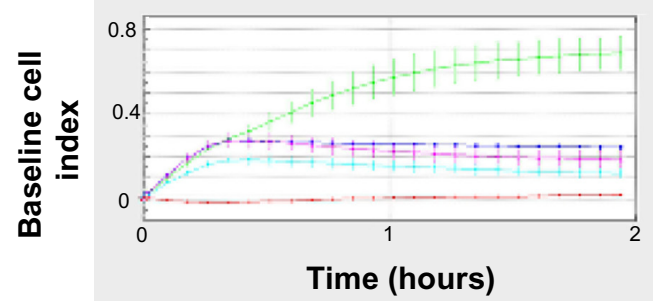

KMH2 (100,000 cells/well)

U937 (100,000 cells/well)
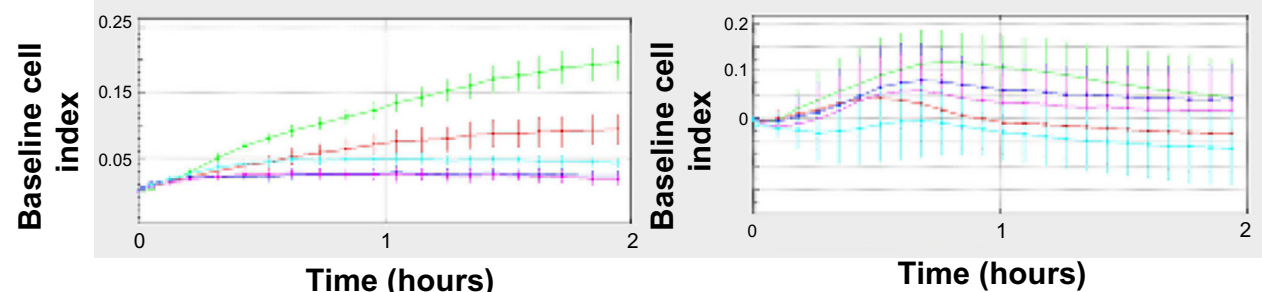

Figure I Fibronectin pre-coating significantly increases Jurkat, $\mathrm{K} 562$, and $\mathrm{KMH} 2$ cell attachment to the bottom of the culture well.

Notes: (A) Fibronectin induced a rapid B- $\mathrm{Cl}$ increase ( $<2$ hours) in Jurkat cells. The maximum $\mathrm{B}-\mathrm{Cl}$ value within 2 hours is included for each substrate. The $\mathrm{B}-\mathrm{Cl}$ is presented as means \pm SD from at least two (up to four) independent wells (calculated by $\times$ CELLigence). (B) Fibronectin unlike other substrates also increased the $\mathrm{B}-\mathrm{Cl}$ for $\mathrm{KMH} 2$ and K562. For U937 cells, not even the fibronectin succeeded in attaching leukemia cells to the bottom of the well.

Abbreviations: $\mathrm{B}-\mathrm{Cl}$, baseline cell index; SD, standard deviation.

L1236 cells attached and spread onto the surface of the well plate (cell attaching stage). After this cell attaching stage, we observed a stabilization phase (up to 15-18 hours), in which the B-CI did not increase. For Jurkat cells, after 15-18 hours, the B-CI began to increase in a lightweight manner, suggesting a cell growth stage. Unlike the Jurkat cell line, L1236 did not show a cell growth stage after 15-18 hours. This fact may be linked with the lowest division rate of this cell line. When Jurkat or L1236 cells are grown in culture plates, they tend to group together, forming cell 


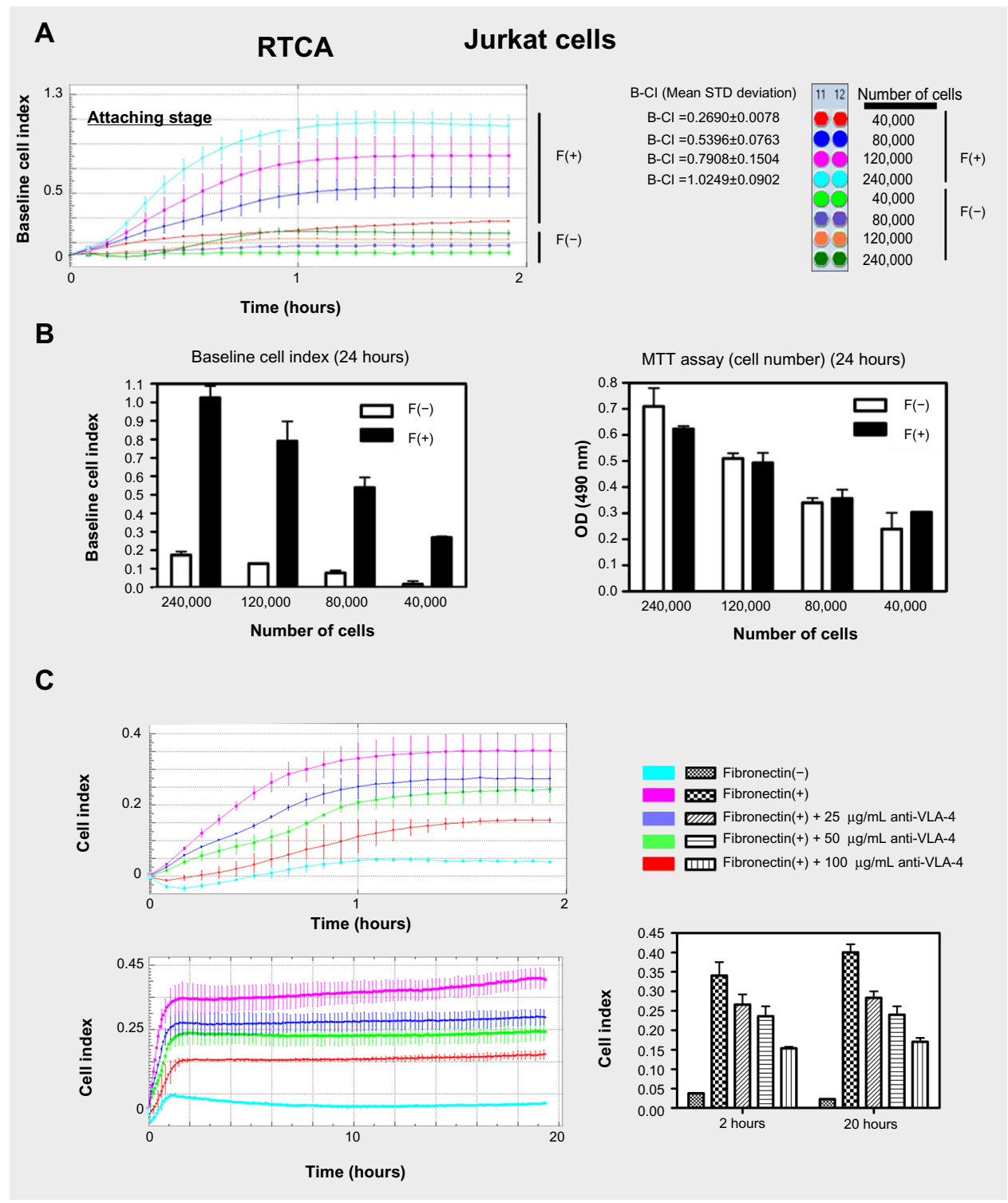

Figure 2 Increases in the number of cells seeded into the wells, correlated with increases in $\mathrm{Cl}$.

Notes: (A) We seeded different numbers of Jurkat cells $(40,000,80,000,120,000$, and 240,000 cells/well) in the presence $(\mathrm{F}+)$ or absence (F-) of fibronectin. We observed in the RTCA graph the correlation between the B-Cl (mean \pm SEM from at least two [up to four] independent wells [2 hours]) and the number of cells seeded (cell-attaching stage). We highlight that in the absence of fibronectin, the values of B-Cl were next to zero. (B) B-Cl versus MTT assay. After 24 hours, we showed that the MTT (mean \pm SEM) values correlated with those obtained in the RTCA (mean \pm SEM) only in the presence of fibronectin (Spearman's rho; $P=0.046$ ). MTT values in the presence or absence of fibronectin were similar. (C) The anti-VLA-4 antibody inhibits Jurkat cells from attaching to fibronectin. Mean $\mathrm{Cl}$ value (mean \pm SEM) for 50,000 cells/well, incubated in the presence of different concentrations of anti-VLA-4.

Abbreviations: $\mathrm{B}-\mathrm{Cl}$, baseline cell index; $\mathrm{Cl}$, cell index; F, fibronectin; MTT, 3-(4,5-dimethylthiazol-2-yl)-2,5-diphenyltetrazolium bromide; RTCA, real-time cell analysis; SEM, standard error of the mean.

clumps, and then later divide, increasing the size of the clumps. As we show in Figure 3, fibronectin pre-coating facilitated the attachment of these cells to the bottom of the E-Plate, inducing a monolayer-type growth. This spatial distribution correlated with the fact that fibronectin is able to efficiently attach the leukemia/lymphoma cells, and thus, cell distribution emulates the typical monolayer growth of an epithelial-like cell culture, adapting the close configuration found in the bone marrow or in the lymph nodes. At the same time, this explains the B-CI increases observed when the cells are seeded in the presence of fibronectin.

\section{Drug cytotoxicity monitoring in the presence or absence of fibronectin: RTCA versus MTT assay}

In order to check if fibronectin allowed the real-time monitoring of the cytotoxic action of several compounds, we incubated Jurkat and L1236 cells with different drugs, such 


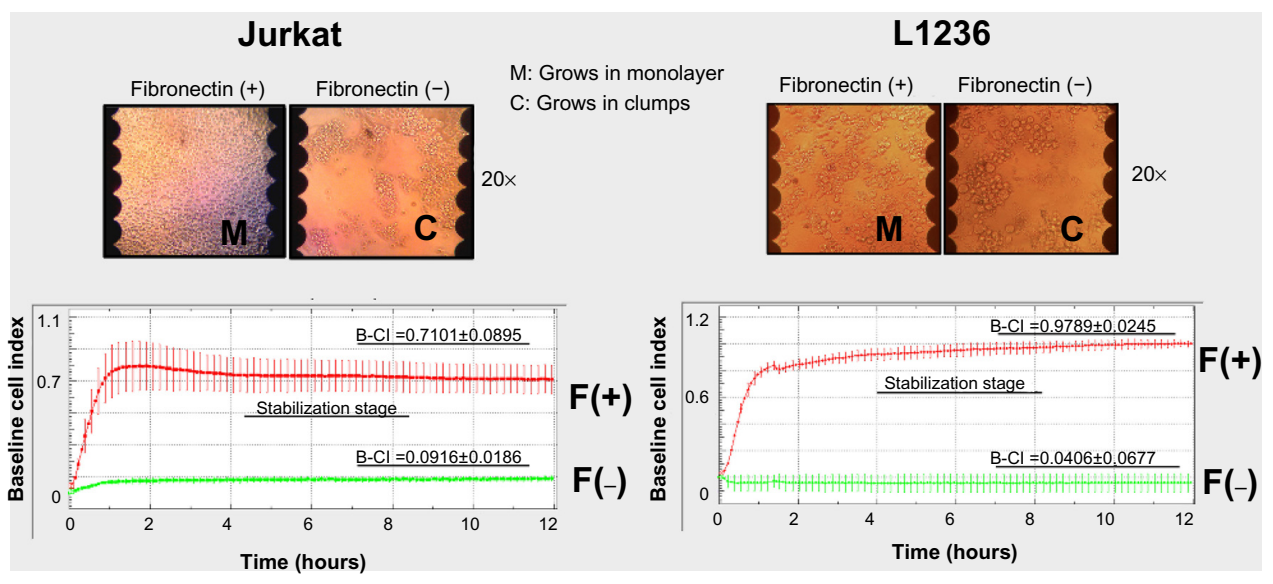

Figure 3 Real-time monitoring of Jurkat/LI236 growth/attachment (leukemia).

Notes: We seeded I20,000 cells (Jurkat/LI236) in duplicate in the presence (F+)/absence (F-) of fibronectin. After the cell-attaching stage, we observed a stabilization stage, in which the $\mathrm{Cl}$ did not increase. We show that fibronectin pre-coating induces Jurkat/LI 236 cell attachment to the bottom of the wells of the E-Plate, inducing a monolayertype growth (M).

Abbreviations: $\mathrm{Cl}$, cell index; $\mathrm{F}$, fibronectin; $\mathrm{B}-\mathrm{Cl}$, baseline cell index.

as FasL $(50 \mathrm{ng} / \mathrm{mL})$, oxaliplatin $(100 \mu \mathrm{M})$, bendamustine $(200 \mu \mathrm{M})$, or trabectedin $(10 \mathrm{nM})$. As seen in Figure 4A, the cell growth and the cytotoxic response were only monitored in the presence of fibronectin. Furthermore, we incubated Jurkat and L1236 cell lines with several chemotherapeutic agents at different drug concentrations: trabectedin $(1,10$, and $100 \mathrm{nM})$, doxorubicin $(0.1,1$, and $10 \mu \mathrm{M})$, cisplatin $(0.1$, $1,10 \mu \mathrm{M}$ ), and gemcitabine $(1,10,100 \mu \mathrm{M}$ ) (up to $24-48$ hours). Once finished the RTCA experiment, we performed an MTT measurement on the same plate. We include in Figure 4B the changes in the percentage values for each dose with respect to the control (non-treated), for both the RTCA and the MTT method. We simultaneously treated Jurkat/L1236 cell lines with the same drug concentrations in a 96-well plate in the absence of fibronectin. In Figure 4B, we show that the results between RTCA and MTT in the presence of fibronectin are comparable. Moreover, we also show that the MTT values are similar when cells are incubated in the absence/presence of fibronectin under these experimental conditions (Figure 4B).

\section{Higher levels of $\mathrm{Cl}$ enable efficient monitoring of cell cytotoxicity}

Finally, to demonstrate the importance of obtaining the highest $\mathrm{CI}$ levels for the proper monitoring of the cytotoxic action of a specific compound, we incubated Jurkat cells in the presence of different substrates. As expected, when cells were attached to fibronectin and higher B-CI levels compared to collagen or gelatin were therefore obtained, we observed significant decreases in the RTCA curves in response to FasL. Decreases in CI value were virtually undetectable when other substrates were used, preventing real-time cell monitoring. We simultaneously performed an MTT assay to compare the data obtained with the RTCA with a conventional method (Figure 5).

\section{Discussion}

The xCELLigence system is a new approach that makes real-time monitoring of adherent cells possible. The basis of its technology relies on the use of culture plates with gold microelectrodes in their base. These electrodes are connected to a computer that records the impedance differences within an electrical circuit. The difference in the electrical signal derived from $\mathrm{xCELLigence} \mathrm{is} \mathrm{the} \mathrm{CI.} \mathrm{Thus,} \mathrm{due} \mathrm{to} \mathrm{an}$ increase in the impedance within the electrical circuit, the greater the number of cells deposited on the bottom of the well, the greater the CI reading is. To date, RTCA technology has mostly been applied to adherent cells. For this reason, it was not useful in the study of cells derived from hematological malignancies. ${ }^{1,2,8-11}$ Recently, one report has described a system for the real-time measuring of cell adhesion in nonadherent cells. ${ }^{4}$ With this in mind, we have adapted the methodology described by others to xCELLigence for monitoring drug toxicity over hematologic cell lines. This strategy is based on the coating of the bottom of cell culture surfaces with specific substrates, some of which are known to facilitate cell attachment in the extracellular matrix (ECM). ${ }^{7,12}$ Several authors have described the interaction between leukemic cell receptor (VLA-4) and stromal fibronectin, ${ }^{6}$ a high molecularweight glycoprotein that is primarily synthesized by fibroblasts in the ECM that binds to membrane receptor proteins called integrins. Moreover, some $\mathrm{T}$ cell acute lymphoblastic 


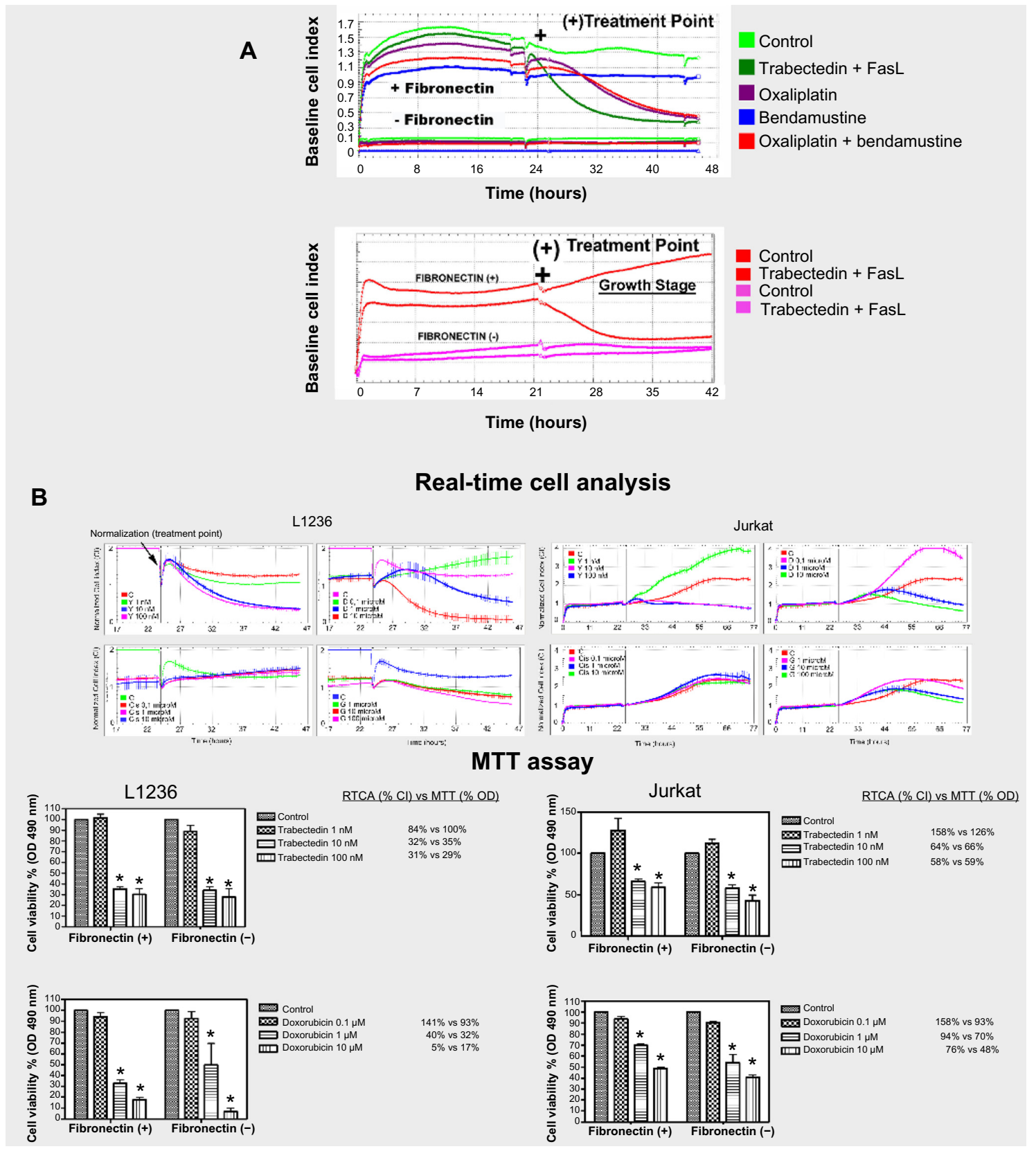

Figure 4 Drug cytotoxicity monitoring of Jurkat/LI236 cells in the presence/absence of Fibronectin: RTCA vs MTT assay.

Notes: (A) We incubated Jurkat/LI236 cells (treatment point $[+])$ in the presence of trabectedin $(T)(10 \mathrm{nM})+$ FasL $(50 \mathrm{ng} / \mathrm{mL})$, bendamustine $(200 \mu M)$, oxaliplatin $(100 \mu \mathrm{M})$, trabectedin $(10 \mathrm{nM})+$ FasL $(50 \mathrm{ng} / \mathrm{mL})$, or bendamustine + oxaliplatin (up to 24 hours). Fibronectin allowed robust monitoring of drug-induced cytotoxicity. Clear decreases of the $\mathrm{B}-\mathrm{Cl}$ correlated with the presence of a large number of apoptotic cells as observed by microscopy (data not shown). (B) RTCA vs MTT: Jurkat/LI236 cells were incubated in the presence of $\mathrm{T}(\mathrm{I}, \mathrm{I}, 0$, and $100 \mathrm{nM}), \mathrm{D}(0 . \mathrm{I}, \mathrm{I}$, and $10 \mu \mathrm{M})$, Cis $(0 . \mathrm{I}, \mathrm{I}$, and I0 $\mu \mathrm{M})$, and $\mathrm{G}$ (I, I0, and I00 $\mu \mathrm{M})$ (up to $24-48$ hours). The presence vs absence of fibronectin did not appear to affect drug-induced toxicity for Jurkat/LI 236 cells using the MTT method, suggesting that fibronectin does not protect Jurkat cells under these experimental conditions.

Abbreviations: B-Cl, baseline cell index; Cis, cisplatin; D, doxorubicin; FasL, Fas ligand; G, gemcitabine; MTT, 3-(4,5-dimethylthiazol-2-yl)-2,5-diphenyltetrazolium bromide; OD, optical density; RTCA, real-time cell analysis; vs, versus.

leukemia (T/-ALL) cell lines (Jurkat, HSB-2, and CEM) or acute myelogenous leukemia cell lines, ${ }^{6}$ attach to collagen and laminin because they also express the correspondent linking receptors on their surface. ${ }^{7,13,14}$ Thus, malignant cells from leukemia or T/B cell lymphoma may bind to these proteins, which results in their capacity to invade tissues rich in ECM, such as lymphatic nodes or the bone marrow. Based on this rationale, we first assayed the relative capacity of different substrates to attach several cells lines such as Jurkat, L1236, KMH2, U937, and K562 onto the surface of the E-Plates. Our data show unequivocal evidence that the presence of fibronectin, a glycoprotein with a key role in cell adhesion, growth, 


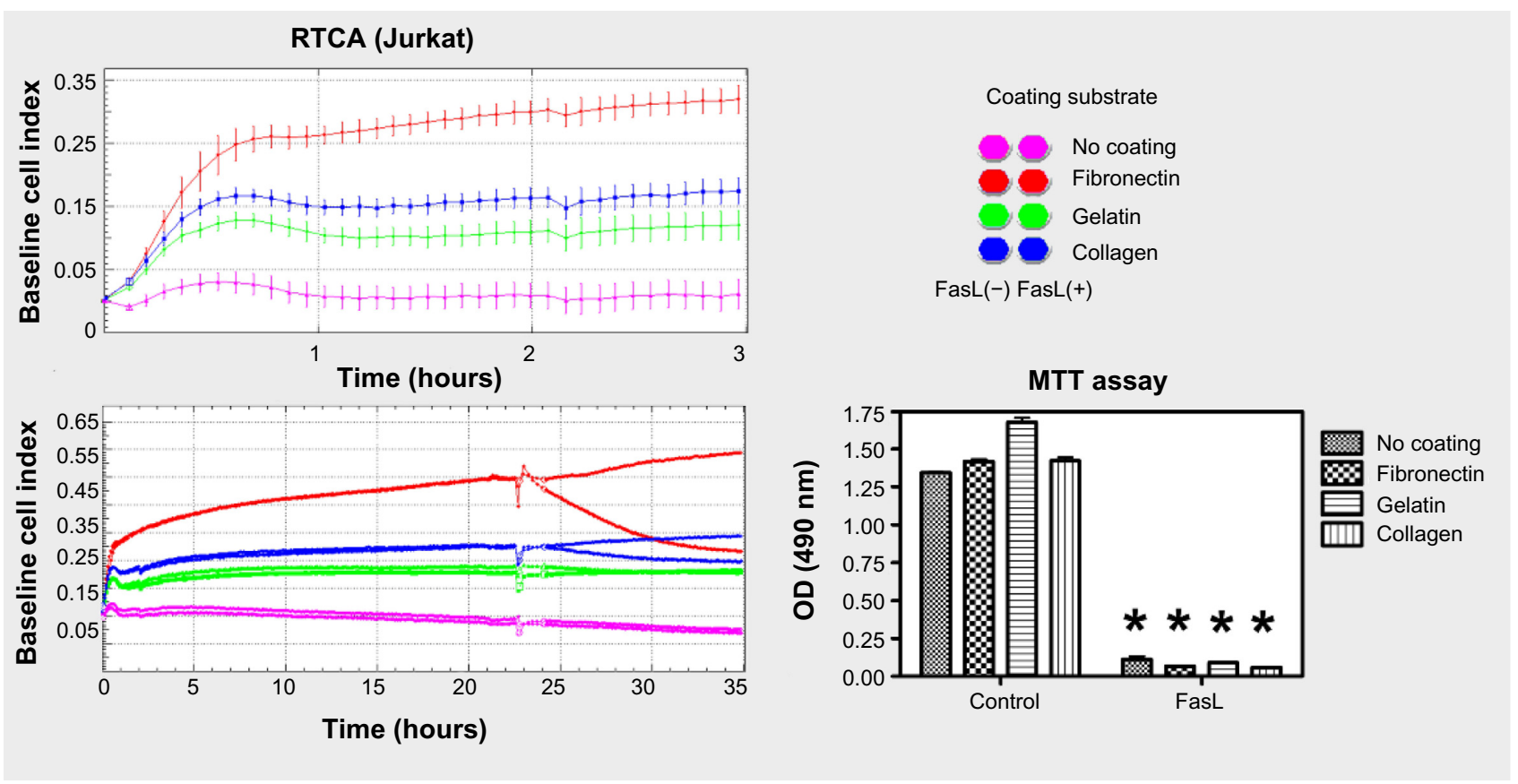

Figure 5 Higher levels of the B-Cl enable efficient monitoring of cell cytotoxicity.

Notes: Jurkat cells were incubated in the presence of the different substrates (fibronectin, collagen, or gelatin) and FasL. Simultaneously, we performed an MTT (mean \pm SEM) assay in order to compare the data obtained with the RTCA with that from a conventional method.

Abbreviations: B-Cl, baseline cell index; FasL, Fas ligand; MTT, 3-(4,5-dimethylthiazol-2-yl)-2,5-diphenyltetrazolium bromide; RTCA, real-time cell analysis; SEM, standard error of the mean; OD, optical density.

migration, and differentiation, significantly facilitates the attaching of almost all the leukemia/lymphoma cells tested when compared to laminin, collagen, or gelatin (Figure 1). ${ }^{15,16}$ As adhesion to fibronectin occurs mainly through the VLA-4 integrin receptor, we incubated Jurkat cells in the presence of different concentrations of anti-VLA-4 antibody. The presence of $100 \mu \mathrm{g} / \mathrm{mL}$ of the anti-VLA-4 inhibited up to $60 \%$ of the CI values obtained when cells were incubated only in the presence of fibronectin (20 hours; Figure 2). Higher concentrations of anti-VLA-4 corresponded with higher blockades of cell attachment to the bottom of the wells. This data clearly suggests the direct implication of this integrin in the adherence of Jurkat cells onto RTCA plates. We also show that thanks to the properties of fibronectin, Jurkat and L1236 leukemia/lymphoma cells are deposited in a monolayer configuration onto the well surface and this event is robustly monitored in real-time by the xCELLigence (CI) (Figure 3). We do not rule out the possibility of additional interactions of fibronectin with any other receptor or even the presence of lipopolysaccharides in the fibronectin solution that facilitates cell-substrate attachment. However, the high degree of attachment inhibition (up to $60 \%$ ) obtained with increased concentrations of anti-VLA-4 demonstrates the specific implication of this receptor. We next showed the feasibility of monitoring the cytotoxic effects of several chemotherapeutic agents on different leukemia/lymphoma cells (Figure 4). We include in Figure 4B the percentage values for each dose with respect to the control (non-treated) for both the RTCA and the MTT method. The percentages obtained from the two methods were comparable. Only at certain non-cytotoxic concentrations ( $1 \mathrm{nM}$ trabectedin L1236 or 1 $\mathrm{nM}$ trabectedin/doxorubicin, Jurkat) we observed significant increases in the CI that did not correspond to significant increases in the MTT values. We attribute this to the fact that, at such low concentrations, the activity of the mitochondrial succinate dehydrogenase is not affected; nevertheless, certain cell morphology parameters are modified, and thus may be monitored, revealing additional data. Finally, we also show that under these experimental conditions, the MTT values obtained when cells are incubated in the absence/presence of fibronectin were similar (Figure 4B). Although not significant, we observed a slight tendency to survive in cells incubated in the presence of fibronectin.

Despite the results showed in this manuscript, many questions remain to be elucidated. These issues are focused on the types of regulations that may influence the ECM elements, such as fibronectin on cell culture behavior (growth, differentiation, etc). We cannot discard this 
interference for other cells lines and/or substrates. Thus, some authors have described in agreement with our data, that the interaction of leukemic cells with fibronectin does not confer significant resistance to chemotherapeutic agents. ${ }^{17}$ On the other hand, there are also studies that show how acute myeloid leukemia and/or ALL-derived blast cells bind to the cellular matrix and ECM components of bone marrow stroma, and may determine the fate of small numbers of leukemic cells surviving after cytotoxic chemotherapy. ${ }^{18}$ In this context, certain elements of the ECM such as fibronectin may play a key role in the activation of the resistance mechanism developed against chemotherapy, generating privileged bone marrow niches for these cells. ${ }^{12,19,20}$ Therefore, we believe that the presence of matrix glycoproteins could provide a better physiological environment for cells. Finally, it is important to highlight that each cell model may exhibit different cell behavior in the presence of several coating substrates. The maximum B-CI reached by two cell lines may be completely different (depending on cell size, receptors, etc). Not all cell lines, as in the case of the U937, will be adaptable to this method. Thus, it is important to carry out a pre-setting stage for each new cell line before starting an experimental procedure. This real-time method may be essential and could also save a considerable amount of time and resources when choosing specific times/dosages for an in-depth study of the molecular events associated with the mechanism of action of a drug. Therefore, in view of these evidences, we consider that this model may represent a very useful tool that will allow us to study the role played by interactions of proteins of the ECM with leukemia/lymphoma cells.

In conclusion, we show the first approach for the realtime monitoring of drug-induced cytotoxicity in nonadherent cell lines derived from hematological malignancies. This time- and cost-saving label-free method is an ideal tool for identifying the optimal time point for further molecular and biochemical analysis of cellular events. As the cell population is continuously monitored, we may record any change or fluctuation in the cells without interference.

\section{Author contributions}

All authors contributed toward data analysis, drafting and revising the paper and agree to be accountable for all aspects of the work.

\section{Acknowledgments}

The authors thank A Font and J Ortiz de Zarate (Roche Diagnostics, Basel, Switzerland) for their valuable technical support. This work was supported by a grant from Mundipharma (Cambridge, UK).

\section{Disclosure}

The authors report no conflicts of interest in this work.

\section{References}

1. Eckhardt BL, Francis PA, Parker BS, Anderson RL. Strategies for the discovery and development of therapies for metastatic breast cancer. Nat Rev Drug Discov. 2012;11(6):479-497.

2. Kustermann S, Boess F, Buness A, et al. A label-free, impedance-based real time assay to identify drug-induced toxicities and differentiate cytostatic from cytotoxic effects. Toxicol In Vitro. 2013;27(5):1589-1595.

3. Limame R, Wouters A, Pauwels B, et al. Comparative analysis of dynamic cell viability, migration and invasion assessments by novel real-time technology and classic endpoint assays. PLoS One. 2012;7(10):e46536.

4. Obr A, Röselová P, Grebeňová D, Kuželová K. Real-time monitoring of hematopoietic cell interaction with fibronectin fragment: the effect of histone deacetylase inhibitors. Cell Adh Migr. 7(3):275-282.

5. Hamawy MM, Oliver C, Mergenhagen SE, Siraganian RP. Adherence of rat basophilic leukemia (RBL-2H3) cells to fibronectin-coated surfaces enhances secretion. J Immunol. 1992;149(2):615-621.

6. Matsunaga T, Takemoto N, Sato T, et al. Interaction between leukemic-cell VLA-4 and stromal fibronectin is a decisive factor for minimal residual disease of acute myelogenous leukemia. Nat Med. 2003;9(9): 1158-1165.

7. Mobley JL, Ennis E, Shimizu Y. Differential activation-dependent regulation of integrin function in cultured human T-leukemic cell lines. Blood. 1994;83(4):1039-1050.

8. Daker M, Ahmad M, Khoo AS. Quercetin-induced inhibition and synergistic activity with cisplatin - a chemotherapeutic strategy for nasopharyngeal carcinoma cells. Cancer Cell Int. 2012;12(1):34.

9. Hanusová V, Králová V, Schröterová L, Trilecová L, Pakostová A, Skálová L. The effectiveness of oracin in enhancing the cytotoxicity of doxorubicin through the inhibition of doxorubicin deactivation in breast cancer MCF7 cells. Xenobiotica. 2010;40(10):681-690.

10. Kumar B, Yadav A, Lang J, Teknos TN, Kumar P. Dysregulation of microRNA-34a expression in head and neck squamous cell carcinoma promotes tumor growth and tumor angiogenesis. PLoS One. 2012;7(5): e37601.

11. Yamashita-Kashima Y, Iijima S, Yorozu K, et al. Pertuzumab in combination with trastuzumab shows significantly enhanced antitumor activity in HER2-positive human gastric cancer xenograft models. Clin Cancer Res. 2011;17(15):5060-5070.

12. Aoudjit F, Vuori K. Integrin signaling in cancer cell survival and chemoresistance. Chemother Res Pract. 2012;2012:283181.

13. Ivanoff J, Talme T, Sundqvist KG. The role of chemokines and extracellular matrix components in the migration of $\mathrm{T}$ lymphocytes into three-dimensional substrata. Immunology. 2005;114(1):53-62.

14. Ricard I, Payet MD, Dupuis G. Clustering the adhesion molecules VLA-4 (CD49d/CD29) in Jurkat T cells or VCAM-1 (CD106) in endothelial (ECV 304) cells activates the phosphoinositide pathway and triggers Ca2+ mobilization. Eur J Immunol. 1997;27(6):1530-1538.

15. Harima A, Nakaseko C, Yokota A, et al. Fibronectin promotes cell proliferation of human pre-B cell line via its interactions with VLA-4 and VLA-5. Hematology. 2008;13(4):236-243.

16. Pankov R, Yamada KM. Fibronectin at a glance. J Cell Sci. 2002; 115(Pt 20):3861-3863.

17. Mudry RE, Fortney JE, York T, Hall BM, Gibson LF. Stromal cells regulate survival of B-lineage leukemic cells during chemotherapy. Blood. 2000;96(5):1926-1932.

18. Bradstock KF, Gottlieb DJ. Interaction of acute leukemia cells with the bone marrow microenvironment: implications for control of minimal residual disease. Leuk Lymphoma. 1995;18(1-2):1-16. 
19. Meads MB, Gatenby RA, Dalton WS. Environment-mediated drug resistance: a major contributor to minimal residual disease. Nat Rev Cancer. 2009;9(9):665-674.
20. Meads MB, Hazlehurst LA, Dalton WS. The bone marrow microenvironment as a tumor sanctuary and contributor to drug resistance. Clin Cancer Res. 2008;14(9):2519-2526.

\section{Publish your work in this journal}

OncoTargets and Therapy is an international, peer-reviewed, open access journal focusing on the pathological basis of all cancers, potential targets for therapy and treatment protocols employed to improve the management of cancer patients. The journal also focuses on the impact of management programs and new therapeutic agents and protocols on

\section{Dovepress}

patient perspectives such as quality of life, adherence and satisfaction. The manuscript management system is completely online and includes a very quick and fair peer-review system, which is all easy to use. Visit http://www.dovepress.com/testimonials.php to read real quotes from published authors.

\footnotetext{
Submit your manuscript here: http://www.dovepress.com/oncotargets-and-therapy-journal
} 